Internat. J. Math. \& Math. Sci.

Vol. 22, No. 3 (1999) 445-458

S 0161-1712<99>22445-3

(c) Electronic Publishing House

\title{
ON THE RITT ORDER AND TYPE OF A CERTAIN CLASS OF FUNCTIONS DEFINED BY BE-DIRICHLETIAN ELEMENTS
}

\section{MARCEL BERLAND}

(Received 2 September 1997)

AвSTRACT. We introduce the notions of Ritt order and type to functions defined by the series

$$
\sum_{n=1}^{\infty} f_{n}\left(\sigma+i \tau_{0}\right) \exp \left(-s \lambda_{n}\right), \quad s=\sigma+i \tau,(\sigma, \tau) \in \mathbf{R} \times \mathbf{R}
$$

indexed by $\boldsymbol{T}_{0}$ on $\mathbf{R}$, where $\left(\lambda_{n}\right)_{1}^{\infty}$ is a $D$-sequence and $\left(f_{n}\right)_{1}^{\infty}$ is a sequence of entire functions of bounded index with at most a finite number of zeros. By definition, the series are $B E$-Dirichletian elements. The notions of order and type of functions, defined by $B$ Dirichletian elements, are considered in [3, 4]. In this paper, using a technique similar to that used by M. Blambert and M. Berland [6], we prove the same properties of Ritt order and type for these functions.

Keywords and phrases. Ritt order and type, entire functions of bounded index, $B E$ Dirichletian elements, Dirichletian elements.

1991 Mathematics Subject Classification. 30A16, 30A64.

\section{Preliminary lemmas}

DEFINITION 1.1 (B. Lepson [10]). An entire function $f$ is said to be of bounded index if there exists a nonnegative integer $v$ such that

$$
\max \left\{\frac{\left|f^{(k)}(s)\right|}{k !} \mid k \in\{0,1, \ldots, v\}\right\} \geq \frac{\left|f^{(j)}(s)\right|}{j !}, \quad\left(f^{(0)}(s)=f(s)\right)
$$

for all $j$ and for all $s$. The least such integer $v$ is called the index of $f$.

THEOREM A (F. Gross [8]). An entire function with at most a finite number of zeros is of bounded index if and only if it is of the form $P(s) \exp (\alpha s)$, where $P(s)$ is polynomial and $\alpha$ is a complex constant.

THEOREM B (S. M. Shah [16]). Let $f(s)=P(s) \exp (\alpha s)$, where $\alpha$ is any complex number and $P(s)$ is a polynomial of degree less than $n$. Then $f$ is of bounded index and the index $v \leq p$, where $p$ is any integer such that $p \geq n-1$ and

$$
\frac{n|\alpha|}{p+1}+\left(\frac{n(n-1)}{2 !}|\alpha|^{2}\right) \frac{1}{p(p+1)}+\cdots+\frac{|\alpha|^{n}}{(p-n+2) \cdots(p+1)} \leq 1 .
$$

Let $\left(\lambda_{n}\right)_{1}^{\infty}$ be a $D$-sequence (that is a positive strictly increasing unbounded sequence) and $\left(f_{n}\right)_{1}^{\infty}$ be a sequence of entire functions $f_{n}$ of bounded index $v_{n}$ with 
at most a finite number of zeros from Theorem A. As a result of the two theorems, we have $\forall s \in \mathbf{C}, \forall n \in \mathbf{N} \backslash\{0\}$

$$
f_{n}(s)=P_{n}(s) \exp \left(\alpha_{n} s\right),
$$

where $P_{n}(s)$ is a polynomial of degree $m_{n}$ and $\alpha_{n}$ is a complex constant, that is,

$$
s \longmapsto P_{n}(s)=\sum_{j=0}^{m_{n}} a_{n, j} s^{j} \quad \text { with } a_{n, m_{n}} \neq 0 \text { and } s \in \mathbf{C} .
$$

Let us suppose that $\exists k \in] 0, \lambda_{1}[, \forall n \in \mathbf{N} \backslash\{0\}$

$$
\alpha_{n} \in \overline{d_{(0, k)}},
$$

where $\overline{d_{(0, k)}}$ is the closed disc centered at 0 and of radius $k$.

Consider the space of elements

$$
\left\{f_{\tau_{0}}\right\}: \sum_{n=1}^{\infty} f_{n}\left(\sigma+i \tau_{0}\right) \exp \left(-s \lambda_{n}\right), \quad s=\sigma+i \tau,(\sigma, \tau) \in \mathbf{R} \times \mathbf{R}
$$

indexed by $\boldsymbol{T}_{0}$ on $\mathbf{R}$. By definition, $\left\{f_{\tau_{0}}\right\}$ is the $B E$-Dirichletian element. Let

$$
\begin{gathered}
\beta=\limsup _{n \rightarrow \infty}\left\{\frac{m_{n}}{\lambda_{n}}\right\}, \\
A_{n}=\max \left\{\left|a_{n j}\right| \mid j \in\left\{0,1, \ldots, m_{n}\right\}\right\} \quad \forall n \in \mathbf{N} \backslash\{0\} .
\end{gathered}
$$

Consider the associated Dirichletian element

$$
\left\{f_{A}\right\}: \sum_{n=1}^{\infty} A_{n} \exp \left(-s \lambda_{n}\right),
$$

whose coefficients are strictly positive and denote, by $\sigma_{c}^{f_{A}}$, the abscissa of convergence of $\left\{f_{A}\right\}$.

Let us state three lemmas due to M. Blambert and M. Berland [6] which we use later. These demonstrations are obvious because this sequence $\left(\alpha_{n}\right)_{1}^{\infty}$ is bounded.

LEMMA 1.1. If $\sigma_{c}^{f_{A}}=-\infty, \beta<\infty, \forall n \in \mathbf{N} \backslash\{0\}, \alpha_{n} \in \overline{d_{(0, k)}}$, then $\left\{f_{\tau_{0}}\right\}$ converges absolutely on $\mathbf{C}$ for any arbitrary $\tau_{0}$ in $\mathbf{R}$.

LEMMA 1.2. If $\sigma_{c}^{f_{A}}=-\infty, \beta<\infty, \forall n \in \mathbf{N} \backslash\{0\}, \alpha_{n} \in \overline{d_{(0, k)}}$, we have $\forall \tau_{0} \in \mathbf{R}, \forall \boldsymbol{T} \in \mathbf{R}$

$$
\lim _{\sigma \rightarrow \infty} f_{\tau_{0}}(\sigma+i \tau)=0
$$

LEMMA 1.3. If $\sigma_{c}^{f_{A}}=-\infty, \forall n \in \mathbf{N} \backslash\{0\}, \alpha_{n} \in \overline{d_{(0, k)}}$, we have $\forall \tau_{0} \in \mathbf{R}, \forall \sigma \in \mathbf{R}$

$$
\begin{aligned}
P_{n}\left(\sigma+i \tau_{0}\right) \exp [ & \left.\alpha_{n}\left(\sigma+i \tau_{0}\right)-\sigma \lambda_{n}\right] \\
= & \lim _{\tau_{2} \rightarrow \infty}\left\{\frac{1}{\tau_{2}} \int_{\tau_{1}}^{\tau_{2}} f_{\tau_{0}}(\sigma+i \tau) \exp \left(i \tau \lambda_{n}\right) d \tau\right\},
\end{aligned}
$$

where $\tau_{1}$ is any arbitrary real number. 
2. Main theorems. Let us define the following quantities. For each $\sigma$ on $\mathbf{C}$,

$$
\begin{aligned}
M\left(\sigma ; f_{\tau_{0}}\right) & =\sup \left\{\left|f_{\tau_{0}}\left(\sigma^{\prime}+i \tau^{\prime}\right)\right| \mid \sigma^{\prime} \geq \sigma, \tau^{\prime} \in \mathbf{R}\right\}, \\
M_{n^{\prime}}\left(\sigma ; f_{\tau_{0}}\right) & =\sup \left\{\left|f_{\tau_{0}, n^{\prime}}\left(\sigma^{\prime}+i \tau^{\prime}\right)\right| \mid \sigma^{\prime} \geq \sigma, \boldsymbol{\tau}^{\prime} \in \mathbf{R}\right\}, \\
\mu\left(\sigma ; f_{\tau_{0}}\right) & =\sup \left\{\left|f_{n}\left(\sigma+i \tau_{0}\right)\right| \exp \left(-\sigma \lambda_{n}\right) \mid n \in \mathbf{N} \backslash\{0\}\right\}, \\
\mu_{n^{\prime}}\left(\sigma ; f_{\tau_{0}}\right) & =\sup \left\{\left|f_{n}\left(\sigma+i \tau_{0}\right)\right| \exp \left(-\sigma \lambda_{n}\right) \mid n \geq n^{\prime}\right\} ;
\end{aligned}
$$

where

$$
f_{\tau_{0}, n^{\prime}}(s)=\sum_{n=n^{\prime}}^{\infty} f_{n}\left(\sigma+i \tau_{0}\right) \exp \left(-s \lambda_{n}\right) .
$$

The quantities defined above are finite.

REMARK. The function $\sigma \mapsto M\left(\sigma ; f_{\tau_{0}}\right)$ is decreasing onto $\mathbf{R}$.

THEOREM 2.1. If $\sigma_{c}^{f_{A}}=-\infty, \beta<\infty, \forall n \in \mathbf{N} \backslash\{0\}, \alpha_{n} \in \overline{d_{(0, k)}}$, we have

$$
\lim _{\sigma \rightarrow \infty}\left\{M\left(\sigma ; f_{\tau_{0}}\right)\right\}=0 \quad \text { and } \quad \lim _{\sigma \rightarrow-\infty}\left\{M\left(\sigma ; f_{\tau_{0}}\right)\right\}=\infty .
$$

Proof. We have $\forall \varepsilon \in] 0,1\left[, \exists n^{\prime}\left(=n_{\varepsilon}^{\prime}\right) \in \mathbf{N} \backslash\{0\}, \forall n \geq n^{\prime}, m_{n} / \lambda_{n}<\beta+\varepsilon\right.$ and $\exists n^{\prime \prime}\left(=n_{\varepsilon}^{\prime \prime}\right) \in \mathbf{N} \backslash\{0\}, \forall n \geq n^{\prime \prime}, k / \lambda_{n}<\varepsilon, \forall \sigma>0$ such that

$$
\begin{aligned}
& \quad \sum_{n=n_{1}\left(=\max \left\{n^{\prime}, n^{\prime \prime}\right\}\right)}^{\infty}\left|f_{n}\left(\sigma+i \tau_{0}\right)\right| \exp \left(-\sigma \lambda_{n}\right) \\
& \leq \sum_{n=n_{1}}^{\infty} A_{n} \exp \left\{-\sigma \lambda_{n}\left[1-\left(\frac{(\beta+\varepsilon) \log \left(1+|\sigma|+\left|\tau_{0}\right|\right)}{\sigma}+\varepsilon\left(1+\frac{\left|\tau_{0}\right|}{\sigma}\right)\right)\right]\right\},
\end{aligned}
$$

$\left.\forall \varepsilon^{\prime} \in\right] 0,1-\varepsilon\left[, \exists \sigma^{\prime}\left(=\sigma_{\varepsilon^{\prime}}\right)>0, \forall \sigma>\sigma^{\prime}\right.$,

$$
\frac{(\beta+\varepsilon) \log \left(1+|\sigma|+\left|\tau_{0}\right|\right)+\varepsilon\left|\tau_{0}\right|}{\sigma}<\varepsilon^{\prime}
$$

and

$$
\begin{aligned}
\sigma\left[1-\left(\frac{(\beta+\varepsilon) \log \left(1+|\sigma|+\left|\boldsymbol{\tau}_{0}\right|\right)+\varepsilon\left|\boldsymbol{\tau}_{0}\right|}{\sigma}+\varepsilon\right)\right] \\
>\sigma\left[(1-\varepsilon)-\varepsilon^{\prime}\right]>\sigma^{\prime}\left[(1-\varepsilon)-\varepsilon^{\prime}\right](>0) .
\end{aligned}
$$

Therefore, $\exists n_{1} \in \mathbf{N} \backslash\{0\}$ such that

$$
M_{n_{1}}\left(\sigma ; f_{\tau_{0}}\right)<f_{A, n_{1}}\left(\sigma^{\prime}\left[(1-\varepsilon)-\varepsilon^{\prime}\right]\right)=\sum_{n=n_{1}}^{\infty} A_{n} \exp \left[-\sigma^{\prime}\left((1-\varepsilon)-\varepsilon^{\prime}\right) \lambda_{n}\right],
$$

where

$$
\lim _{\sigma \rightarrow \infty}\left\{M_{n_{1}}\left(\sigma ; f_{\tau_{0}}\right)\right\}=0
$$


On the other hand, we have, $\forall n \in\left\{1,2, \ldots, n_{1}-1\right\}$

$\lim _{\sigma \rightarrow \infty}\left\{P_{n}\left(\sigma+i \tau_{0}\right) \exp \left[\alpha_{n}\left(\sigma+i \tau_{0}\right)-\sigma \lambda_{n}\right]\right\}=0 \quad\left(\right.$ with $\lambda_{1}>k$ and $\left.\alpha_{n} \in \overline{d_{(0, k)}}\right)$.

We have

$$
\lim _{\sigma \rightarrow \infty}\left\{M\left(\sigma ; f_{\tau_{0}}\right)\right\}=0 .
$$

On the other hand, $\forall \sigma<0$, if $M\left(\sigma ; f_{\tau_{0}}\right)$ is bounded onto $\mathbf{R}$ implies that (from Lemma 1.3)

$$
\forall n \in \mathbf{N} \backslash\{0\}:\left\{j \in\left\{0,1, \ldots, m_{n}\right\} \Longrightarrow a_{n, j}=0\right\} .
$$

Or, thus, we get the contradiction that

$$
a_{n, m_{n}} \neq 0 \quad \forall n \in \mathbf{N} \backslash\{0\}
$$

and

$$
\lim _{\sigma \rightarrow-\infty}\left\{M\left(\sigma ; f_{\tau_{0}}\right)\right\}=\infty .
$$

Thus, (2.13) and (2.16) prove the theorem.

Furthermore, let

$$
\begin{aligned}
\rho_{R}^{f_{\tau_{0}}} & =\limsup _{\sigma \rightarrow-\infty}\left\{\frac{\log ^{+}\left(\log ^{+}\left(M\left(\sigma ; f_{\tau_{0}}\right)\right)\right)}{-\sigma}\right\}, \\
\lambda_{R}^{f_{\tau_{0}}} & =\liminf _{\sigma \rightarrow-\infty}\left\{\frac{\log ^{+}\left(\log ^{+}\left(M\left(\sigma ; f_{\tau_{0}}\right)\right)\right)}{-\sigma}\right\} .
\end{aligned}
$$

By definition, $\rho_{R}^{f_{\tau_{0}}}$ and $\lambda_{R}^{f_{\tau_{0}}}$ are the Ritt-order and the lower Ritt-order of function $f_{\tau_{0}}$ defined by $B E$-Dirichletian element $\left\{f_{\tau_{0}}\right\}$. Also, $M\left(\sigma ; f_{A}\right)$ is defined in a similar manner with $f_{A}$ in the place of $f_{\tau_{0}}$. It is trivial that

$$
\begin{aligned}
& \rho_{R}^{f_{A}}=\limsup _{\sigma \rightarrow-\infty}\left\{\frac{\log ^{+}\left(\log ^{+}\left(f_{A}(\sigma)\right)\right)}{-\sigma}\right\}, \\
& \lambda_{R}^{f_{A}}=\liminf _{\sigma \rightarrow-\infty}\left\{\frac{\log ^{+}\left(\log ^{+}\left(f_{A}(\sigma)\right)\right)}{-\sigma}\right\} .
\end{aligned}
$$

THEOREM 2.2. If $\sigma_{c}^{f_{A}}=-\infty, \beta<\infty, \forall n \in \mathbf{N} \backslash\{0\}, \alpha_{n} \in \overline{d_{(0, k)}}$, and $L\left(=\limsup _{n \rightarrow \infty}\{\log n /\right.$ $\left.\left.\lambda_{n}\right\}\right)<\infty$, we have $\forall \boldsymbol{T}_{0} \in \mathbf{R}$,

$$
\rho_{R}^{f_{\tau_{0}}}=\rho_{R}^{f_{A}} \quad \text { and } \quad \lambda_{R}^{f_{\tau_{0}}}=\lambda_{R}^{f_{A}} .
$$

Proof. (1) We get the inequalities, $\forall \tau_{0} \in \mathbf{R}$,

$$
\rho_{R}^{f_{A}} \leq \rho_{R}^{f_{\tau_{0}}} \quad \text { and } \quad \lambda_{R}^{f_{A}} \leq \lambda_{R}^{f_{\tau_{0}}} .
$$

$\tau_{0}$ is any arbitrary real number. Consider the closed interval $I(s, \lambda)=\left\{s^{\prime} \in \mathbf{C}|| \sigma^{\prime}-\right.$ $\left.\sigma \mid \leq \lambda>0, \tau^{\prime}=\tau_{0}\right\}$, where $\sigma^{\prime}=\operatorname{Re}\left(s^{\prime}\right), \tau^{\prime}=\operatorname{Im}\left(s^{\prime}\right)$, and $s=\sigma+i \tau_{0}$. Let $\forall n \in \mathbf{N} \backslash\{0\}$, 
ON THE RITT ORDER AND TYPE OF A CERTAIN CLASS OF FUNCTIONS ...

$$
\begin{aligned}
& p_{n}(s, \lambda)=\sup \left\{\left|P_{n}\left(s^{\prime}\right)\right| \mid s^{\prime} \in \overline{d_{(s, \lambda)}}\right\}, \\
& p_{n}^{*}(s, \lambda)=\sup \left\{\left|P_{n}\left(s^{\prime}\right)\right| \mid s^{\prime} \in I(s, \lambda)\right\} .
\end{aligned}
$$

Using Lemma 1.3, we have $\forall \sigma^{\prime} \in[\sigma-\lambda, \sigma+\lambda], \forall n \in \mathbf{N} \backslash\{0\}$,

$$
\left|P_{n}\left(\sigma^{\prime}+i \tau_{0}\right)\right| \exp \left[\left(\operatorname{Re}\left(\alpha_{n}\right)-\lambda_{n}\right) \sigma^{\prime}-\operatorname{Im}\left(\alpha_{n}\right) \tau_{0}\right] \leq M\left(\sigma-\lambda ; f_{\tau_{0}}\right),
$$

and then (M. Blambert and M. Berland [6])

$$
\begin{gathered}
p_{n}^{*}(s, \lambda) \exp \left[-(\sigma+\lambda)\left(\lambda_{n}-\operatorname{Re}\left(\alpha_{n}\right)\right)-\operatorname{Im}\left(\alpha_{n}\right) \tau_{0}\right] \leq M\left(\sigma-\lambda ; f_{\tau_{0}}\right), \\
6^{-m_{n}} p_{n}(s, \lambda) \leq p_{n}^{*}(s, \lambda), \\
A_{n}(1+|s|)^{-m_{n}} \leq p_{n}(s, \lambda) \quad \forall \lambda \geq 1, \quad \text { (M. Berland [1]). }
\end{gathered}
$$

Therefore, we have $\forall \lambda \geq 1, \forall \sigma \in \mathbf{R}, \forall n \in \mathbf{N} \backslash\{0\}$

$$
A_{n} \leq M\left(\sigma-\lambda ; f_{\tau_{0}}\right) \exp \left\{\left[\sigma+\lambda+\frac{m_{n}}{\lambda_{n}^{\prime}} \log \left(6\left(1+|\sigma|+\left|\tau_{0}\right|\right)\right)+\frac{\operatorname{Im}\left(\alpha_{n}\right)}{\lambda_{n}^{\prime}} \tau_{0}\right] \lambda_{n}^{\prime}\right\},
$$

where $\lambda_{n}^{\prime}=\lambda_{n}-\operatorname{Re}\left(\alpha_{n}\right)$.

We have $\forall \varepsilon \in] 0,1\left[, \exists n_{1} \in \mathbf{N} \backslash\{0\}, \forall n \geq n_{1}\right.$

$$
A_{n} \leq M\left(\sigma-\lambda ; f_{\tau_{0}}\right) \exp \left\{\left[\sigma+\lambda+\left(\beta^{\prime}+\varepsilon\right) \log \left(6\left(1+|\sigma|+\left|\tau_{0}\right|\right)\right)+\varepsilon\right] \lambda_{n}^{\prime}\right\},
$$

and $\left.\forall \varepsilon_{1} \in\right] 0,1\left[, \exists \sigma_{1}\left(=\sigma_{\varepsilon_{1}}\right)>0, \forall \sigma<-\sigma_{1}\right.$

$$
\frac{\lambda+\left(\beta^{\prime}+\varepsilon\right) \log \left(6\left(1+|\sigma|+\left|\tau_{0}\right|\right)\right)+\varepsilon}{-\sigma}<\varepsilon_{1},
$$

where $\beta^{\prime}=\limsup _{n \rightarrow \infty}\left\{m_{n} / \lambda_{n}^{\prime}\right\}(<\infty)$ which implies that, $\left.\forall \varepsilon_{1} \in\right] 0,1\left[, \forall n \geq n_{1}, \forall \sigma<\sigma_{1}\right.$

$$
A_{n} \leq M\left(\sigma-\lambda ; f_{\tau_{0}}\right) \exp \left[\sigma\left(1-\varepsilon_{1}\right) \lambda_{n}^{\prime}\right]
$$

Now, $\forall n \in \mathbf{N} \backslash\{0\}, \operatorname{Re}\left(\alpha_{n}\right) \leq k$ (because $\alpha_{n} \in \overline{d_{(0, k)}}$ )

$$
\lambda_{n}^{\prime}=\lambda_{n}\left(1-\frac{\operatorname{Re}\left(\alpha_{n}\right)}{\lambda_{n}}\right) \geq \lambda_{n}\left(1-\frac{k}{\lambda_{n}}\right)
$$

We have, $\left.\forall \varepsilon_{2} \in\right] 0,1\left[, \exists n^{\prime} \in \mathbf{N} \backslash\{0\}, \forall n \geq n^{\prime}\right.$,

$$
\frac{k}{\lambda_{n}}<\varepsilon_{2} \quad \text { and } \quad \lambda_{n}^{\prime} \geq \lambda_{n}\left(1-\varepsilon_{2}\right) \quad\left(\Longrightarrow \beta^{\prime}=\beta\right)
$$

which implies that, $\forall \lambda \geq 1, \forall n \geq \max \left\{n_{1}, n^{\prime}\right\}\left(=n_{2}\right), \forall \sigma<-\sigma_{1}$

$$
A_{n} \exp \left[-\sigma\left(1-\varepsilon_{1}\right)\left(1-\varepsilon_{2}\right) \lambda_{n}\right] \leq M\left(\sigma-\lambda ; f_{\tau_{0}}\right)
$$

Put, $\forall n \in \mathbf{N} \backslash\{0\}$

$$
\mu_{n_{(1,2)}}=\left(1-\varepsilon_{1}\right)\left(1-\varepsilon_{2}\right) \lambda_{n} \quad(>0),
$$


$\left(\mu_{n_{(1,2)}}\right)$ is a $D$-sequence. Consider the Dirichletian element

$$
\left\{f_{A_{(1,2)}}\right\}: \sum_{n=1}^{\infty} A_{n} \exp \left(-s \mu_{n_{(1,2)}}\right)
$$

indexed by the couple $(1,2)$ and denote, by $\sigma_{\mathcal{C}}^{f_{A_{(1,2)}}}$, the abscissa of convergence of $\left\{f_{A_{(1,2)}}\right\}$. We have, $\sigma_{c}^{f_{A_{(1,2)}}}=-\infty$ (since $\left.\sigma_{c}^{f_{A}}=-\infty\right), f_{A_{(1,2)}}$ is an entire function and, its Ritt-order is

$$
\rho_{R}^{f_{A_{(1,2)}}}=\limsup _{\sigma \rightarrow-\infty}\left\{\frac{\log ^{+}\left(\log ^{+}\left(f_{A_{(1,2)}}(\sigma)\right)\right)}{-\sigma}\right\},
$$

and its lower Ritt-order is

$$
\lambda_{R}^{f_{A_{(1,2)}}}=\liminf _{\sigma \rightarrow-\infty}\left\{\frac{\log ^{+}\left(\log ^{+}\left(f_{A_{(1,2)}}(\sigma)\right)\right)}{-\sigma}\right\} .
$$

Now, $\forall \sigma \in \mathbf{R}$,

$$
f_{A_{(1,2)}}(\sigma)=f_{A}\left(\sigma\left(1-\varepsilon_{1}\right)\left(1-\varepsilon_{2}\right)\right)
$$

and

$$
\begin{aligned}
& \rho_{R}^{f_{A_{(1,2)}}}=\left(1-\varepsilon_{1}\right)\left(1-\varepsilon_{2}\right) \rho_{R}^{f_{A}}, \\
& \lambda_{R}^{f_{A_{(1,2)}}}=\left(1-\varepsilon_{1}\right)\left(1-\varepsilon_{2}\right) \lambda_{R}^{f_{A}} .
\end{aligned}
$$

Put (Q. S. Liu [11]) $\forall \sigma \in \mathbf{R}$,

$$
\mu_{n_{2}}\left(\sigma ; f_{A_{(1,2)}}\right)=\sup \left\{A_{n} \exp \left(-\sigma \mu_{n_{(1,2)}}\right) \mid n \geq n_{2}\right\} .
$$

We have $\forall \varepsilon>0, \forall \sigma \in \mathbf{R}$

$$
\begin{aligned}
f_{A_{(1,2), n_{2}}}(\sigma) & \leq \sum_{n=n_{2}}^{\infty}\left(A_{n} \exp \left[-(\sigma-L-\varepsilon) \mu_{n_{(1,2)}}\right]\right) \exp \left[-(L+\varepsilon) \mu_{n_{(1,2)}}\right] \\
& \leq \mu_{n_{2}}\left(\sigma-L-\varepsilon ; f_{A_{(1,2)}}\right) K_{n_{2}}(\varepsilon)
\end{aligned}
$$

with $K_{n_{2}}(\varepsilon)=\sum_{n=n_{2}}^{\infty} \exp \left[-(L+\varepsilon) \mu_{n_{(1,2)}}\right]$.

Hence, $\forall \varepsilon>0, \forall \sigma \in \mathbf{R}$

$$
f_{A_{(1,2), n_{2}}}(\sigma) \leq \mu_{n_{2}}\left(\sigma-L-\varepsilon ; f_{A_{(1,2)}}\right) K_{n_{2}}(\varepsilon) .
$$

Then we have, $\forall \lambda \geq 1, \exists n_{2}=\max \left\{n_{1}, n^{\prime}\right\}, \forall n \geq n_{2}, \forall \sigma<-\sigma_{1}$

$$
A_{n} \exp \left(-\sigma \mu_{n_{(1,2)}}\right) \leq M\left(\sigma-\lambda ; f_{\tau_{0}}\right) \text {. }
$$

This implies that

$$
\mu_{n_{2}}\left(\sigma ; f_{A_{(1,2)}}\right) \leq M\left(\sigma-\lambda ; f_{\tau_{0}}\right)
$$

From (2.45) and (2.47), we have

$$
\begin{aligned}
& f_{A_{(1,2), n_{2}}}(\sigma+L+\varepsilon) \leq M\left(\sigma-\lambda ; f_{\tau_{0}}\right) K_{n_{2}}(\varepsilon), \\
& f_{R}^{f_{A_{(1,2), n_{2}}}} \leq \rho_{R}^{f_{\tau_{0}}}\left(\lim _{\sigma \rightarrow-\infty}\left(\frac{\sigma-\lambda}{\sigma+L+\varepsilon}\right)\right)=\rho_{R}^{f_{\tau_{0}}} .
\end{aligned}
$$


Or

$$
\rho_{R}^{f_{A_{(1,2), n_{2}}}}=\rho_{R}^{f_{A_{(1,2)}}} \quad \text { (M. Blambert [5]). }
$$

We have, $\left.\forall \varepsilon_{1} \in\right] 0,1\left[, \forall \varepsilon_{2} \in\right] 0,1[$

$$
\begin{aligned}
& \rho_{R}^{f_{A_{(1,2)}}}=\left(1-\varepsilon_{1}\right)\left(1-\varepsilon_{2}\right) \rho_{R}^{f_{A}} \leq \rho_{R}^{f_{\tau_{0}}}, \\
& \lambda_{R}^{f_{A_{(1,2)}}}=\left(1-\varepsilon_{1}\right)\left(1-\varepsilon_{2}\right) \lambda_{R}^{f_{A}} \leq \lambda_{R}^{f_{\tau_{0}}} .
\end{aligned}
$$

As $\varepsilon_{1}$ and $\varepsilon_{2}$ are arbitrary, we have

$$
\rho_{R}^{f_{A}} \leq \rho_{R}^{f_{\tau_{0}}}, \text { and then } \lambda_{R}^{f_{A}} \leq \lambda_{R}^{f_{\tau_{0}}} .
$$

(2) We get the inequalities, $\forall \tau_{0} \in \mathbf{R}$,

$$
\rho_{R}^{f_{\tau_{0}}} \leq \rho_{R}^{f_{A}} \quad \text { and } \quad \lambda_{R}^{f_{\tau_{0}}} \leq \lambda_{R}^{f_{A}} .
$$

From Theorem 2.1, we have $\forall \varepsilon \in] 0,1\left[, \forall \varepsilon^{\prime} \in\right] 0,1-\varepsilon\left[, \exists \sigma^{\prime}>0, \forall \sigma<-\sigma^{\prime}\right.$

$$
\begin{aligned}
\sigma[1-((\beta+\varepsilon) & \left.\left.\frac{\log \left(1+|\sigma|+\left|\tau_{0}\right|\right)}{|\sigma|}+\varepsilon\left(1+\frac{\left|\tau_{0}\right|}{|\sigma|}\right)\right) \theta_{\sigma}\right] \\
& =\sigma\left[1+(\beta+\varepsilon) \frac{\log \left(1+|\sigma|+\left|\tau_{0}\right|\right)}{|\sigma|}+\varepsilon\left(1+\frac{\left|\tau_{0}\right|}{|\sigma|}\right)\right] \\
& >\sigma\left[1+\left((\beta+2 \varepsilon) \varepsilon^{\prime}+\varepsilon\right)\right]=\sigma\left(1+\varepsilon_{1}\right),
\end{aligned}
$$

where $\varepsilon_{1}=(\beta+2 \varepsilon) \varepsilon^{\prime}+\varepsilon, \theta_{\sigma}=1$ if $\sigma>0$ and $\theta_{\sigma}=-1$ if $\sigma<0$.

Hence, $\forall \sigma<-\sigma^{\prime}, \exists n_{1} \in \mathbf{N} \backslash\{0\}$,

$$
M_{n_{1}}\left(\sigma ; f_{\tau_{0}}\right) \leq f_{A, n_{1}}\left(\sigma\left(1+\varepsilon_{1}\right)\right),
$$

which implies that

$$
\rho_{R}^{f_{\tau_{0}, n_{1}}} \leq \rho_{R}^{f_{A, n_{1}}}\left(1+\varepsilon_{1}\right)=\left(1+\varepsilon_{1}\right) \rho_{R}^{f_{A}},
$$

and where

$$
\rho_{R}^{f_{\tau_{0}, n_{1}}} \leq \rho_{R}^{f_{A}} \quad \text { and } \quad \lambda_{R}^{f_{\tau_{0}, n_{1}}} \leq \lambda_{R}^{f_{A}} .
$$

Now, $\sigma \in \mathbf{R}$,

$$
M\left(\sigma ; f_{\tau_{0}}\right) \leq M_{n_{1}}^{0}\left(\sigma ; f_{\tau_{0}}\right)+M_{n_{1}}\left(\sigma ; f_{\tau_{0}}\right),
$$

where

$$
M_{n_{1}}^{0}\left(\sigma ; f_{\tau_{0}}\right)=\sup \left\{\left|f_{\tau_{0}, n_{1}}^{0}\left(\sigma^{\prime}+i \tau^{\prime}\right)\right| \mid \sigma^{\prime} \geq \sigma, \tau^{\prime} \in \mathbf{R}\right\}
$$

and

$$
\left\{f_{\tau_{0}, n_{1}}^{0}\right\}: \sum_{n=1}^{n_{1}-1} f_{n}\left(\sigma+i \tau_{0}\right) \exp \left(-s \lambda_{n}\right) .
$$

Then $\forall \tau_{0} \in \mathbf{R}$,

$$
\rho_{R}^{f_{\tau_{0}}} \leq \max \left\{\rho_{R}^{f_{\tau_{0}, n_{1}}}, \rho_{R}^{f_{T_{0}, n_{1}}^{0}}\right\}=\rho_{R}^{f_{\tau_{0}, n_{1}}}
$$

since $\rho_{R}^{f_{T_{0}, n_{1}}^{0}}=0$. 
Finally, we have

$$
\rho_{R}^{f_{\tau_{0}}} \leq \rho_{R}^{f_{A}}
$$

and, similarly, we can show that

$$
\lambda_{R}^{f_{\tau_{0}}} \leq \lambda_{R}^{f_{A}} .
$$

Hence, (1) and (2) implies (2.21) which proves this theorem.

If $\rho_{R}^{f_{\tau_{0}}}>0$, we put

$$
\begin{gathered}
\tau_{R}^{f_{\tau_{0}}}=\limsup _{\sigma \rightarrow-\infty}\left\{\frac{\log \left(M\left(\sigma ; f_{\tau_{0}}\right)\right)}{\exp \left(-\sigma \rho_{R}^{f_{\tau_{0}}}\right)}\right\}, \\
\nu_{R}^{f_{\tau_{0}}}=\liminf _{\sigma \rightarrow-\infty}\left\{\frac{\log \left(M\left(\sigma ; f_{\tau_{0}}\right)\right)}{\exp \left(-\sigma \rho_{R}^{f_{\tau_{0}}}\right)}\right\} .
\end{gathered}
$$

By definition, $\tau_{R}^{f_{\tau_{0}}}$ and $v_{R}^{f_{\tau_{0}}}$ are the Ritt-type and the lower Ritt-type of order of $f_{\tau_{0}}$. It is trivial that if $\rho_{R}^{f_{A}}>0$,

$$
\begin{gathered}
\tau_{R}^{f_{A}}=\limsup _{\sigma \rightarrow-\infty}\left\{\frac{\log \left(f_{A}(\sigma)\right)}{\exp \left(-\sigma \rho_{R}^{f_{A}}\right)}\right\}, \\
\nu_{R}^{f_{A}}=\liminf _{\sigma \rightarrow-\infty}\left\{\frac{\log \left(f_{A}(\sigma)\right)}{\exp \left(-\sigma \rho_{R}^{f_{A}}\right)}\right\} .
\end{gathered}
$$

THEOREM 2.3. If $\sigma_{c}^{f_{A}}=-\infty, \beta<\infty, L\left(=\lim _{n \rightarrow \infty}\left(\log n / \lambda_{n}\right)\right)=0, \forall n \in \mathbf{N} \backslash\{0\}, \alpha_{n} \in$ $\overline{d_{(0, k)}}, \rho_{R}^{f_{\tau_{0}}}>0$, we have $\forall \tau_{0} \in \mathbf{R}$,

$$
\tau_{R}^{f_{\tau_{0}}}=\tau_{R}^{f_{A}} .
$$

Proof. (1) We have the inequality, $\forall \tau_{0} \in \mathbf{R}$,

$$
\tau_{R}^{f_{A}} \leq \tau_{R}^{f_{\tau_{0}}},
$$

$\tau_{0}$ is any arbitrary real number. From Theorem 2.2, we have, $\left.\forall \varepsilon \in\right] 0,1\left[, \exists n_{1} \in \mathbf{N} \backslash\{0\}\right.$, $\forall n \geq n_{1}, \forall \sigma \in \mathbf{R} ; \forall \lambda \geq 1$,

$$
A_{n} \leq M\left(\sigma-\lambda ; f_{\tau_{0}}\right) \exp \left\{\left[\sigma+\lambda+\left(\beta^{\prime}+\varepsilon\right) \log \left(6\left(1+|\sigma|+\left|\tau_{0}\right|\right)\right)+\varepsilon\right] \lambda_{n}^{\prime}\right\},
$$

where

$$
\lambda_{n}^{\prime}=\lambda_{n}-\operatorname{Re}\left(\alpha_{n}\right) \quad \text { and } \quad \beta^{\prime}=\limsup _{n \rightarrow \infty}\left\{\frac{m_{n}}{\lambda_{n}^{\prime}}\right\} \quad\left(\beta^{\prime}=\beta\right)<\infty .
$$

Now, $\forall \sigma<0$,

$$
A_{n} \leq M\left(\sigma-\lambda ; f_{\tau_{0}}\right) \exp \left\{\left[\sigma-\lambda-\frac{\sigma\left(2 \lambda+(\beta+\varepsilon) \log \left(6\left(1+|\sigma|+\left|\tau_{0}\right|\right)\right)+\varepsilon\right)}{-\sigma}\right] \lambda_{n}^{\prime}\right\} .
$$


Also, we have $\left.\forall \varepsilon_{1} \in\right] 0,1\left[, \exists \sigma_{1}\left(=\sigma_{\varepsilon_{1}}\right)>0, \forall \sigma<-\sigma_{1}\right.$,

$$
\begin{gathered}
\frac{2 \lambda(\beta+\varepsilon) \log \left(6\left(1+|\sigma|+\left|\tau_{0}\right|\right)\right)+\varepsilon}{-\sigma}<\varepsilon_{1}, \\
A_{n} \leq M\left(\sigma-\lambda ; f_{\tau_{0}}\right) \exp \left\{\left[\sigma\left(1-\varepsilon_{1}\right)-\lambda\right] \lambda_{n}^{\prime}\right\} \\
\quad \leq M\left(\sigma-\lambda ; f_{\tau_{0}}\right) \exp \left[\left(\sigma-\frac{\lambda}{1-\varepsilon_{1}}\right) \mu_{n_{(1,2)}}\right],
\end{gathered}
$$

where $\forall n \geq n_{2}=\max \left\{n_{1}, n\right\}$,

$$
\mu_{n_{(1,2)}}=\left(1-\varepsilon_{1}\right)\left(1-\varepsilon_{2}\right) \lambda_{n}, \quad \lambda_{n}^{\prime} \geq \lambda_{n}\left(1-\varepsilon_{2}\right) \quad\left(\varepsilon_{2} \in\right] 0,1[)
$$

which implies that, $\forall \lambda \geq 1, \forall n \geq n_{2}, \forall \sigma<-\sigma_{1}$

$$
A_{n} \leq M\left(\sigma-\lambda ; f_{\tau_{0}}\right) \exp \left[\sigma\left(1-\frac{\lambda}{1-\varepsilon_{1}}\right) \mu_{n_{(1,2)}}\right] .
$$

$\tau_{R}^{f_{\tau_{0}}}$ is the Ritt-type of order of $f_{\tau_{0}}$, we have, $\forall \varepsilon^{\prime}>0, \exists \sigma^{\prime}\left(=\sigma_{\varepsilon^{\prime}}\right)>0, \forall \sigma<-\sigma^{\prime}$

$$
\log \left(M\left(\sigma-\lambda ; f_{\tau_{0}}\right)\right) \leq\left(\tau_{R}^{f_{\tau_{0}}}+\varepsilon^{\prime}\right) \exp \left[-(\sigma-\lambda) \rho_{R}^{f_{\tau_{0}}}\right] .
$$

Hence, $\forall n \geq n_{2}, \forall \varepsilon^{\prime}>0$,

$$
\log A_{n} \leq\left(\tau_{R}^{f_{\tau_{0}}}+\varepsilon^{\prime}\right) \exp \left[-(\sigma-\lambda) \rho_{R}^{f_{\tau_{0}}}\right]+\left(\sigma-\frac{\lambda}{\left(1-\varepsilon_{1}\right)}\right) \mu_{n_{(1,2)}} .
$$

Let us consider $f_{n}$, the function defined by

$$
f_{n}(\sigma)=a \exp [-(\sigma-\lambda) b]+\mu_{n_{(1,2)}}(\sigma+c),
$$

and indexed by $n>n_{2}$. Choosing

$$
a=\tau_{R}^{f_{\tau_{0}}}+\varepsilon^{\prime}>0, \quad b=\rho_{R}^{f_{\tau_{0}}}>0, \quad c=\frac{\lambda}{\varepsilon_{1}-1},
$$

we get $\forall n \geq n_{2}, \forall \sigma \in \mathbf{R} \backslash\left\{\sigma_{n}\right\}$,

$$
f_{n}(\sigma)>f_{n}\left(\sigma_{n}\right)
$$

with

$$
\sigma_{n}-\lambda=\frac{1}{b} \log \left(\frac{a b}{\mu_{n_{(1,2)}}}\right)
$$

and

$$
\begin{gathered}
\lim _{n \rightarrow \infty} \sigma_{n}=-\infty \Longrightarrow \exists n_{3} \in \mathbf{N} \backslash\{0\}, \quad \forall n \geq \max \left\{n_{2}, n_{3}\right\}, \\
\sigma_{n}<-\max \left\{\sigma_{1}, \sigma^{\prime}\right\},
\end{gathered}
$$


where

$$
\begin{aligned}
\log A_{n} \leq f_{n}\left(\sigma_{n}\right) & =\frac{\mu_{n_{(1,2)}}}{b}+\left(\frac{\varepsilon_{1} \lambda}{1-\varepsilon_{1}}+\frac{1}{b} \log \frac{a b}{\mu_{n_{(1,2)}}}\right) \mu_{n_{(1,2)}} \\
& \Uparrow \\
\mu_{n_{(1,2)}}\left(A_{n}^{b / \mu_{n_{(1,2)}}}\right) & \leq e a b \exp \left(\frac{\varepsilon_{1} \lambda b}{1-\varepsilon_{1}}\right) .
\end{aligned}
$$

Or, if $L\left(=\lim _{n \rightarrow \infty}\left(\log n / \lambda_{n}\right)\right)=0$, we have

$$
\tau_{R}^{f_{A_{(1,2)}}} e \rho_{R}^{f_{A_{(1,2)}}}=\limsup _{n \rightarrow \infty}\left\{\mu_{n_{(1,2)}}\left(A_{n}^{f_{R}^{\rho_{A_{(1,2)}} / \mu_{n_{(1,2)}}}}\right)\right\},
$$

(M. Berland [3], following the theorem of Lindelöf-Blambert-Yu) and

$$
\begin{gathered}
\rho_{R}^{f_{\tau_{0}}}=\rho_{R}^{f_{A}}=\frac{1}{\left(1-\varepsilon_{1}\right)\left(1-\varepsilon_{2}\right)} \rho_{R}^{f_{A_{(1,2)}}}, \quad \text { (Theorem 2.2), } \\
\tau_{R}^{f_{A}=\tau_{R}^{f_{A(1,2)}},}
\end{gathered}
$$

from which

$$
\left(1-\varepsilon_{1}\right)\left(1-\varepsilon_{2}\right) \lambda_{n}\left(A_{n}^{\rho_{R}^{f_{A}} /\left[\left(1-\varepsilon_{1}\right)\left(1-\varepsilon_{2}\right) \lambda_{n}\right]}\right) \leq e\left(\tau_{R}^{f_{\tau_{0}}}+\varepsilon^{\prime}\right) \rho_{R}^{f_{A}} \exp \left(\frac{\varepsilon_{1} \lambda \rho_{R}^{f_{\tau_{0}}}}{1-\varepsilon_{1}}\right)
$$

and

$$
A_{n}^{\rho_{R}^{f_{A}} / \lambda_{n}} \leq A_{n}^{\rho_{R}^{f_{A}} /\left[\left(1-\varepsilon_{1}\right)\left(1-\varepsilon_{2}\right) \lambda_{n}\right]} .
$$

Then, $\left.\forall \varepsilon_{1} \in\right] 0,1\left[, \forall \varepsilon_{2} \in\right] 0,1\left[, \forall \varepsilon^{\prime}>0, \rho_{R}^{f_{A}}>0\right.$,

$$
\tau_{R}^{f_{A}} \leq \frac{\tau_{R}^{f_{\tau_{0}}}+\varepsilon^{\prime}}{\left(1-\varepsilon_{1}\right)\left(1-\varepsilon_{2}\right)} \exp \left(\frac{\varepsilon_{1} \lambda \rho_{R}^{f_{\tau_{0}}}}{1-\varepsilon_{1}}\right),
$$

as $\varepsilon_{1}, \varepsilon_{2}$, and $\varepsilon^{\prime}$ are arbitrary, we deduce immediately that

$$
\forall \tau_{0} \in \mathbf{R}: \tau_{R}^{f_{A}} \leq \tau_{R}^{f_{\tau_{0}}} .
$$

(2) We get, when $\tau_{0}$ is a fixed real number, $\forall \varepsilon>0, \exists \sigma_{\varepsilon}>0, \forall \sigma<-\sigma_{\varepsilon}$,

$$
\left|f_{\tau_{0}}(\sigma+i \tau)\right| \leq\left|f_{A}(\sigma(1+\varepsilon))\right| .
$$

In particular, $\left.\forall \varepsilon^{\prime}>0, \exists \sigma_{\varepsilon^{\prime}}>0, \forall \varepsilon \in\right] 0, \varepsilon^{\prime} / \sigma_{\varepsilon^{\prime}}\left[, \exists \sigma_{\varepsilon}>0, \forall \sigma<-\max \left\{\sigma_{\varepsilon}, \sigma_{\varepsilon^{\prime}}\right\}\right.$

$$
f_{A}(\sigma(1+\varepsilon)) \leq f_{A}\left(\sigma-\varepsilon^{\prime}\right), \quad(\text { M. Berland [3] })
$$

and, hence, $\forall \varepsilon^{\prime}>0$,

$$
\forall \sigma<-\max \left\{\sigma_{\varepsilon}, \sigma_{\varepsilon^{\prime}}\right\}: M\left(\sigma ; f_{\tau_{0}}\right) \leq f_{A}\left(\sigma-\varepsilon^{\prime}\right) .
$$


From $\rho_{R}^{f_{\tau_{0}}}=\rho_{R}^{f_{A}}>0$, we get the inequality, $\forall \varepsilon^{\prime}>0$,

$$
\tau_{R}^{f_{\tau_{0}}} \leq \tau_{R}^{f_{A}} \exp \left(\varepsilon^{\prime} \rho_{R}^{f_{A}}\right)
$$

As $\varepsilon^{\prime}$ is arbitrary, we have

$$
\forall \tau_{0} \in \mathbf{R}: \tau_{R}^{f_{\tau_{0}}} \leq \tau_{R}^{f_{A}}
$$

As a result of this theorem, we have an expression for $\tau_{R}^{f_{\tau_{0}}}$ in terms of $\lambda_{n}$ and $A_{n}$.

If $\sigma_{c}^{f_{A}}=-\infty, \beta<\infty, \forall n \in \mathbf{N} \backslash\{0\}, \alpha_{n} \in \overline{d_{(0, k)}}, \forall \tau_{0} \in \mathbf{R}$,

$$
L\left(=\lim _{n \rightarrow \infty}\left(\frac{\log n}{\lambda_{n}}\right)\right)=0, \quad \rho_{R}^{f_{\tau_{0}}}>0,
$$

we have

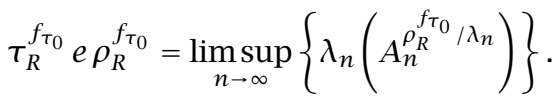

REMARK. The notions of Ritt-type of order of functions, defined by $B$-Dirichletian elements, are considered in [3] with the same result of this theorem.

THEOREM 2.4. If $\sigma_{c}^{f_{A}}=-\infty, \beta<\infty, \forall n \in \mathbf{N} \backslash\{0\}, \alpha_{n} \in \overline{d_{(0, k)}}, L=0, \lambda_{n} \sim \lambda_{n+1}, \varphi$ defined by

$$
\varphi(n)=\frac{\log \left(A_{n} / A_{n+1}\right)}{\lambda_{n+1}-\lambda_{n}},
$$

is a nondecreasing function of $n \geq n_{1}$, and $\rho_{R}^{f_{A}}>0$, we have, $\forall \tau_{0} \in \mathbf{R}$,

$$
v_{R}^{f_{\tau_{0}}}=v_{R}^{f_{A}} .
$$

Proof. (1) We have the inequality, $\forall \tau_{0} \in \mathbf{R}$,

$$
v_{R}^{f_{A}} \leq v_{R}^{f_{\tau_{0}}} .
$$

Suppose that the inequality is false. Then

$$
\exists \tau_{0} \in \mathbf{R}: v_{R}^{f_{\tau_{0}}}<v_{R}^{f_{A}} .
$$

Let $\varepsilon \in] 0, v_{R}^{f_{A}}-v_{R}^{f_{\tau_{0}}}\left[, \varepsilon^{\prime} \in\right] 0, \varepsilon / v_{B}^{f_{A}}\left[\right.$ and $v=v_{R}^{f_{A-\varepsilon}} /\left(1-\varepsilon^{\prime}\right)$; then $v_{R}^{f_{\tau_{0}}}<v<v_{R}^{f_{A}}$. Under the conditions stated in Theorem 2.4, R. K. Srivastava [17] proved that

$$
v_{R}^{f_{A}} e \rho_{R}^{f_{A}}=\liminf _{n \rightarrow \infty}\left\{\lambda_{n}\left(A_{n}^{\rho_{R}^{f_{A}} / \lambda_{n}}\right)\right\},
$$

which implies that $\exists n^{\prime} \in \mathbf{N} \backslash\{0\}, \forall n \geq n^{\prime}$,

$$
v e \rho_{R}^{f_{A}}<\lambda_{n}\left(A_{n}^{\rho_{R}^{f_{A}} / \lambda_{n}}\right) .
$$


Now, $\left.\forall \varepsilon_{1} \in\right] 0,1\left[, \forall \varepsilon_{2} \in\right] 0,1\left[, \exists \sigma_{1}\left(=\sigma_{\varepsilon_{1}}\right)>0, \forall \sigma<-\sigma_{1}, \forall n \geq n_{2}\left(=\max \left\{n_{1}, n^{\prime}\right\}\right)\right.$,

$$
A_{n} \leq M\left(\sigma-\lambda ; f_{\tau_{0}}\right) \exp \left[\left(\sigma-\frac{\lambda}{1-\varepsilon_{1}}\right) \mu_{n_{(1,2)}}\right],
$$

where $\lambda$ is a constant lying in $[1, \infty[$ and

$$
\mu_{n_{(1,2)}}=\left(1-\varepsilon_{1}\right)\left(1-\varepsilon_{2}\right) \lambda_{n} \quad \text { (see Theorem 2.3), }
$$

which gives

$$
\begin{aligned}
& \log A_{n}-\left(\sigma-\frac{\lambda}{1-\varepsilon_{1}}\right) \mu_{n_{(1,2)}} \leq \log \left(M\left(\sigma-\lambda ; f_{\tau_{0}}\right)\right), \\
& \log A_{n}+\frac{\lambda}{1-\varepsilon_{1}} \mu_{n_{(1,2)}}-\sigma \mu_{n_{(1,2)}} \leq \log \left(M\left(\sigma-\lambda ; f_{\tau_{0}}\right)\right) .
\end{aligned}
$$

Let us consider $\varphi_{n}$, the function defined by

$$
\varphi_{n}(\sigma)=\frac{\alpha_{n}-\beta_{n} \sigma}{\exp \left[-(\sigma-\lambda) \rho_{R}^{f_{A}}\right]},
$$

and indexed by $n \geq n_{2}$. Choose

$$
\alpha_{n}=\log A_{n}+\frac{\lambda}{1-\varepsilon_{1}} \mu_{n_{(1,2)}}, \quad \beta_{n}=\mu_{n_{(1,2)}} \quad(>0) .
$$

This takes the maximum value at

$$
\begin{aligned}
& \sigma_{n}=\frac{\alpha_{n}}{\beta_{n}}-\frac{1}{\rho_{R}^{f_{A}}} \quad\left(=\frac{\log A_{n}}{\mu_{n_{(1,2)}}}+\frac{\lambda}{1-\varepsilon_{1}}-\frac{1}{\rho_{R}^{f_{A}}}\right), \\
& \max \left\{\varphi_{n}(\sigma) \mid \sigma \in \mathbf{R}\right\}=\frac{\mu_{n_{(1,2)}}}{\rho_{R}^{f_{A}} e}\left(A^{\rho_{R}^{f_{A}} / \mu_{n_{(1,2)}}}\right) \exp \left(\frac{\varepsilon_{1} \lambda}{1-\varepsilon_{1}} \rho_{R}^{f_{A}}\right) \\
& \leq \frac{\log \left(M\left(\sigma_{n}-\lambda ; f_{\tau_{0}}\right)\right)}{\exp \left[-\left(\sigma_{n}-\lambda\right) \rho_{R}^{f_{\tau_{0}}}\right]} \quad\left(\text { for } \rho_{R}^{f_{A}}=\rho_{R}^{f_{\tau_{0}}}\right)
\end{aligned}
$$

As $\forall n \in \mathbf{N} \backslash\{0\}$,

$$
\mu_{n_{(1,2)}}<\lambda_{n} \Longleftrightarrow A^{\rho_{R}^{f_{A}} / \mu_{n(1,2)}}>A_{n}^{\rho_{R}^{f_{A}} / \lambda_{n}}
$$

which gives

$$
\frac{\left(1-\varepsilon_{1}\right)\left(1-\varepsilon_{2}\right)}{\rho_{R}^{f_{A}} e} \exp \left(\frac{\varepsilon_{1} \lambda}{1-\varepsilon_{1}} \rho_{R}^{f_{A}}\right) \lambda_{n}\left(A_{n}^{\rho_{A}^{f_{A} / \lambda_{n}}}\right) \leq \frac{\log M\left(\sigma_{n}-\lambda ; f_{\tau_{0}}\right)}{\exp \left[\left(-\sigma_{n}-\lambda\right) \rho_{R}^{f_{\tau_{0}}}\right]} .
$$

Finally, we have, $\forall \varepsilon_{3}>0, \exists\left(\sigma_{n}\right)_{1}^{\infty}, \lim _{n \rightarrow \infty} \sigma_{n}=-\infty$,

$$
\frac{\log M\left(\sigma_{n}-\lambda ; f_{\tau_{0}}\right)}{\exp \left[\left(-\sigma_{n}-\lambda\right) \rho_{R}^{f_{\tau_{0}}}\right]} \leq v_{R}^{f_{\tau_{0}}}+\varepsilon_{3}
$$


Hence, we get, $\left.\forall \varepsilon_{3}>0, \forall \varepsilon_{1} \in\right] 0,1\left[, \forall \varepsilon_{2} \in\right] 0,1[$,

$$
\left(\left(1-\varepsilon_{1}\right)\left(1-\varepsilon_{2}\right) \exp \left(\frac{\varepsilon_{1} \lambda}{1-\varepsilon_{1}} \rho_{R}^{f_{A}}\right)\right) v \leq v_{R}^{f_{\tau_{0}}}+\varepsilon_{3} .
$$

Choosing $\varepsilon_{3}=\varepsilon_{1}=\varepsilon_{2}$ of $] 0,1[$, we get

$$
v \leq v_{R}^{f_{\tau_{0}}} .
$$

Thus, we get the contradiction that

$$
v_{R}^{f_{\tau_{0}}}<(v \leq) v_{R}^{f_{\tau_{0}}}
$$

which proves, under the stated conditions, that it is impossible to find a $\boldsymbol{\tau}_{0}$ of $\mathbf{R}$ such that $v_{R}^{f_{\tau_{0}}}<v_{R}^{f_{A}}$.

(2) We have the inequality, $\forall \tau_{0} \in \mathbf{R}$,

$$
v_{R}^{f_{\tau_{0}}} \leq v_{R}^{f_{A}} \quad \text { (see Theorem 2.3, 2). }
$$

As a result of this theorem, we have an expression for $v_{R}^{f_{\tau_{0}}}$ in terms of $\lambda_{n}$ and $A_{n}$, $\forall \tau_{0} \in \mathbf{R}$

$$
v_{R}^{f_{\tau_{0}}} e \rho_{R}^{f_{\tau_{0}}}=\liminf _{n \rightarrow \infty}\left\{\lambda_{n}\left(A_{n}^{\rho_{R}^{f_{\tau_{0}}} / \lambda_{n}}\right)\right\} .
$$

\section{REFERENCES}

[1] M. Berland, Capacité logarithmique et propriétés de minoration des polynômes, 94e Congrès National des Sociétés Savantes, vol. 2, Bibliothèque Nationale, 1969, pp. 21-40.

[2] _ Sur la convergence de certaines séries d'exponentielles dont les coefficients sont des polynômes tayloriens, Thèse, Grenoble, 1983, 1-100.

[3] _ Sur la notion de type de l'ordre à la Ritt de fonctions entières définies par des éléments L-Dirichlétiens, J. Analysis 3 (1995), 17-41. MR 96e:30064. Zbl 868.30004.

[4] - On the lower type of order of functions defined by B-and L-Dirichletian elements, J. Analysis 4 (1996), 125-142. MR 97k:30003. Zbl 869.30003.

[5] M. Blambert, Sur la comparaison des ordres borélien et $(R)$ des fonctions entières d'une certaine classe, Bull. Sci. Math. (2) 89 (1965), 103-125. MR 33\#4274. Zbl 137.27001.

[6] M. Blambert and M. Berland, Sur l'ordre $(R)$ de certaines classes d'applications, Bull. Sci. Math. (2) 98 (1974), no. 1, 63-80. MR 55 8328. Zbl 299.30008.

[7] M. Blambert and R. Parvatham, On the lower order of entire functions defined by LDirichletian elements, Ann. Sci. Math. Québec 3 (1979), no. 2, 199-213 (French). MR 80j:30007. Zbl 425.30021.

[8] F. Gross, Entire functions of bounded index, Proc. Amer. Math. Soc. 18 (1967), 974-980. MR 36\#1649. Zbl 185.14101.

[9] W. K. Hayman, Differential inequalities and local valency, Pacific J. Math. 44 (1973), 117137. MR 47 5240. Zbl 248.30026.

[10] B. Lepson, Differential equations of infinite order, hyperdirichlet series and entire functions of bounded index, Entire Functions and Related Parts of Analysis (Proc. Sympos. Pure Math., LaJolla, Calif.) (Providence, RI), Amer. Math. Soc., 1968, pp. 298-307. MR 38\#6069. Zbl 199.12902. 
[11] Q. S. Liu, On the growth of entire functions defined by B-Dirichletian elements, J. Wuhan Univ. Natur. Sci. Ed. 4 (1989), 1-6 (Chinese).

[12] J. J. MacDonnell, Some convergence theorems for Dirichlet-type series whose coefficients are entire functions of bounded index, D. C. (1957), 1-41.

[13] S. Mandelbrojt, Séries de Dirichlet. Principes et méthodes, Monographies Internationales de Mathematiques Modernes, vol. 11, Gauthier-Villars, Paris, 1969. MR 41\#3721. Zbl 207.07201.

[14] R. Parvatham and J. Thangamani, On the Ritt order of a certain class of functions, Internat. J. Math. Math. Sci. 6 (1983), no. 1, 181-187. MR 84c:30047. Zbl 506.30017.

[15] Q. I. Rahman, On the lower order of entire functions defined by Dirichlet series, Quart. J. Math. Oxford Ser. (2) 7 (1956), 96-99. MR 20\#5282. Zbl 074.29901.

[16] S. M. Shah, Entire functions of bounded index, Proc. Amer. Math. Soc. 19 (1968), 10171022. MR 38\#6070. Zbl 164.08601.

[17] R. K. Srivastava, On the coefficients of an integral function represented by Dirichlet series of finite order, Ganita 13 (1962), 25-35. MR 29\#2372. Zbl 122.31701.

BERLAND: 4, RUE DUPIN, 75006 PARIs, FRANCE

E-mail address: ber1and@emai 1 . enst. fr 


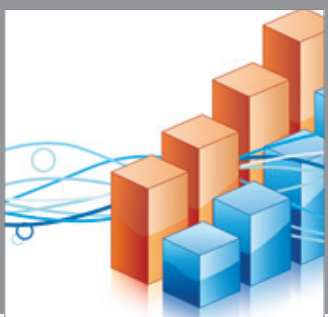

Advances in

Operations Research

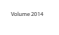

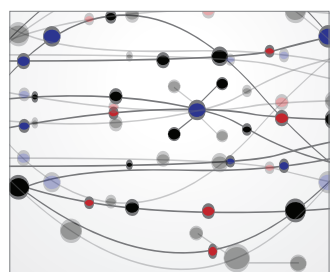

\section{The Scientific} World Journal
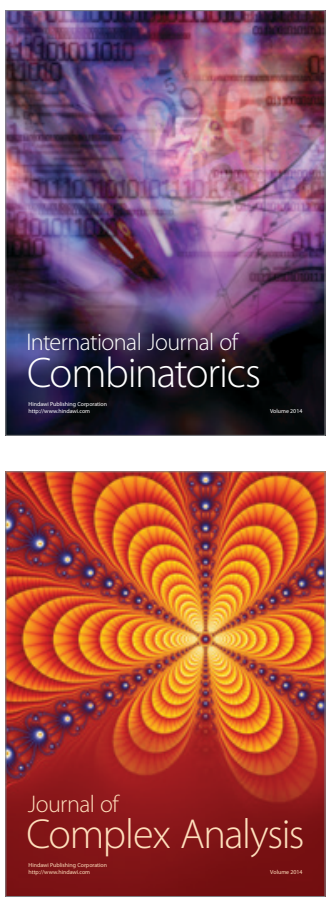

International Journal of

Mathematics and

Mathematical

Sciences
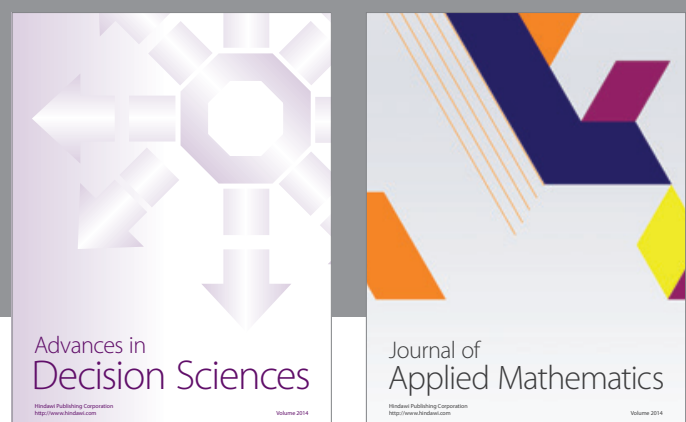

Journal of

Applied Mathematics
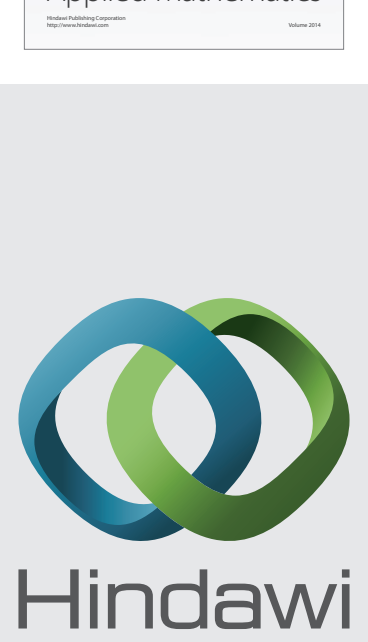

Submit your manuscripts at http://www.hindawi.com
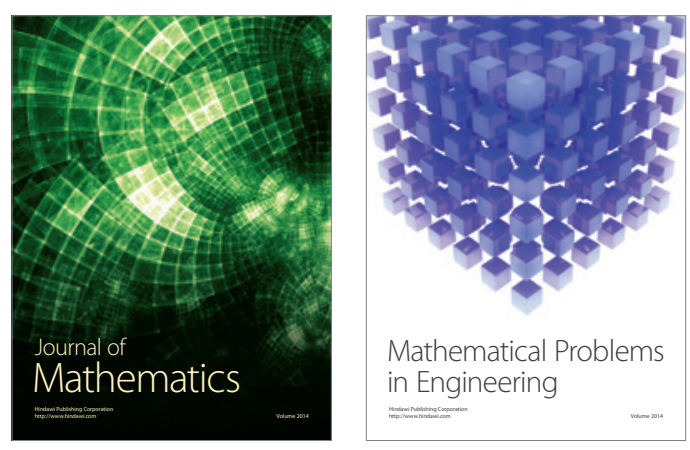

Mathematical Problems in Engineering
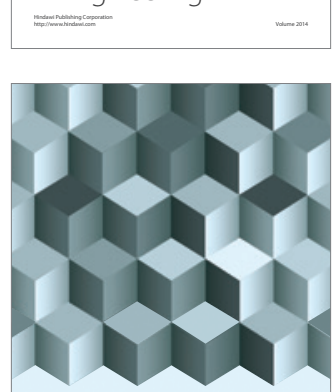

Journal of

Function Spaces
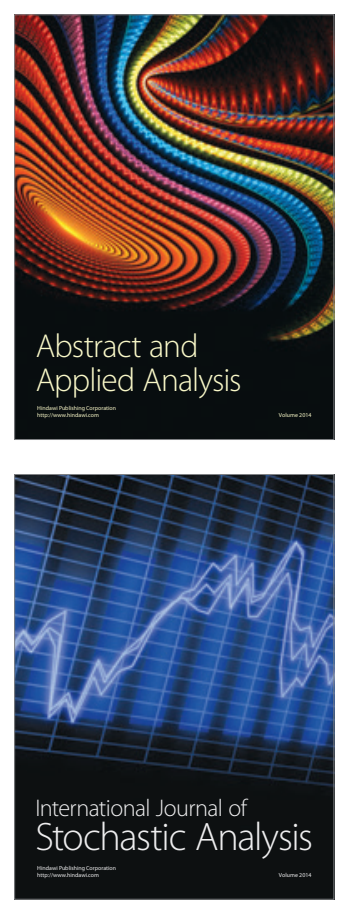

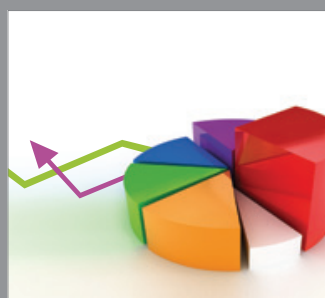

ournal of

Probability and Statistics

Promensencen
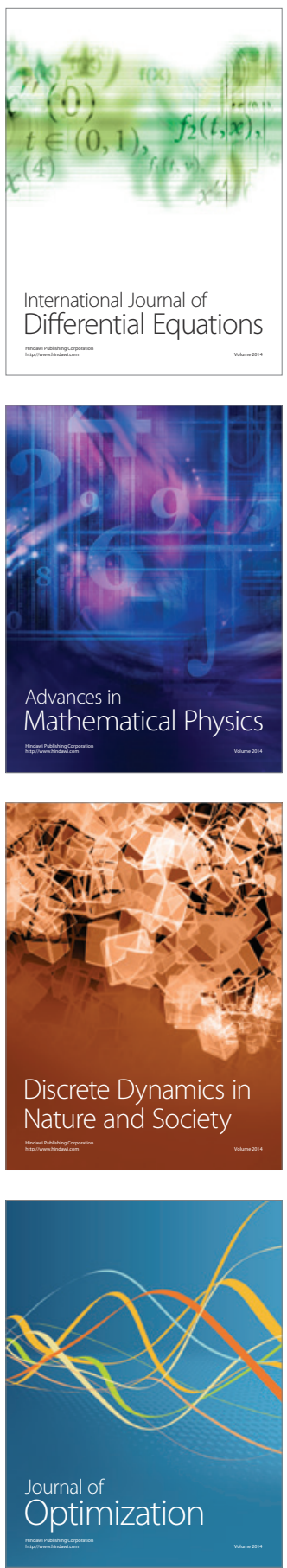